Kastresana A, Sanmamed MF, Wolchok JD. Agonist antibodies to TNFR molecules that costimulate T and NK cells. Clin Cancer Res. 2013;19(5):1044-1053.

12. Melero I, et al. Monoclonal antibodies against the 4-1BB T-cell activation molecule eradicate established tumors. Nat Med. 1997;3(6):682-685.

13. Sznol M, et al. Phase I study of BMS-663513, a fully human anti-CD137 agonist monoclonal antibody, in patients with advanced cancer. J Clin Oncol. 2008;26(15S):3007.

14. Dubrot J, et al. Treatment with anti-CD137 mAbs causes intense accumulations of liver $\mathrm{T}$ cells without selective antitumor immunotherapeutic effects in this organ. Cancer Immunol Immunother. 2010;59(8):1223-1233

15. Seo Y, et al. Cetuximab-mediated ADCC activity is correlated with the cell surface expression level of EGFR but not with the KRAS/BRAF mutational status in colorectal cancer. Oncol Rep. 2014;31(5):2115-2122.

16. Quesnelle KM, Wheeler SE, Ratay MK, Grandis JR. Preclinical modeling of EGFR inhibitor resistance in head and neck cancer. Cancer Biol Ther. 2012;13(10):935-945.

17. Yamashita M, Chattopadhyay S, Fensterl V, Saikia P, Wetzel JL, Sen GC. Epidermal growth factor receptor is essential for Toll-like receptor 3 signaling. Sci Signal. 2012;5(233):ra50.

18. Kumai $T$, et al. EGFR inhibitors augment antitumour helper T-cell responses of HER family-specific immunotherapy. BrJ Cancer. 2013;109(8):2155-2166.

19. Shay $T$, et al. Conservation and divergence in the transcriptional programs of the human and mouse immune systems. Proc Natl Acad Sci U S A. 2013;110(8):2946-2951.

20. Shultz LD, Brehm MA, Garcia-Martinez JV, Greiner DL. Humanized mice for immune system investigation: progress, promise and challenges. Nat Rev Immunol. 2012;12(11):786-798.

\title{
Sex, drugs, and trial design: sex influences the heart and drug responses
}

\author{
Elizabeth Murphy ${ }^{1}$ and Charles Steenbergen ${ }^{2}$ \\ ${ }^{1}$ National Heart, Lung, and Blood Institute, NIH, Bethesda, Maryland, USA. \\ 2Department of Pathology, The Johns Hopkins Medical Institutions, Baltimore, Maryland, USA.
}

\begin{abstract}
Preclinical studies indicate that the phosphodiesterase 5 (PDE5) inhibitor sildenafil is protective against hypertrophy-induced cardiac remodeling. Despite an initial clinical study demonstrating sildenafil-dependent amelioration of pathological remodeling, the cardioprotective effect of this drug was not significant in a large placebo-controlled clinical trail. In this issue, Sasaki and colleagues reveal that the efficacy of PDE5 inhibition in female mice requires estrogen. Induction of cardiac stress in male and intact female mice resulted in increased activation of protein kinase G (PKG) signaling, which was further enhanced by sildenafil. PKG activity was not enhanced in ovariectomized (OVX) female mice as a result of cardiac stress, but administration of estrogen restored PKG activation and enhancement by sildenafil. These data highlight the importance of considering sex-specific differences and drug responses in clinical trial design.
\end{abstract}

\section{Regulation of cGMP levels in the heart}

An increase in nitric oxide (NO) or activation of natriuretic peptide triggers guanylyl cyclase (sGC), resulting in the generation of cGMP, which in turn promotes protein kinase $\mathrm{G}(\mathrm{PKG})$ signaling to regulate multiple cellular processes, including the cardiac response to stress. In addition to sGC, phosphodiesterases (PDEs) regulate cGMP levels via degradation (see Figure 1). While many PDEs are promiscuous and can degrade either cAMP or cGMP (1), PDE5 is reported to specifically degrade cGMP and is inhibited by the drug sildenafil. Under basal conditions, levels of PDE 5 are low in the heart; therefore, PDE5 inhibition with sildenafil has

Conflict of interest: The authors have declared that no conflict of interest exists.

Citation for this article: J Clin Invest. 2014; 124(6):2375-2377. doi:10.1172/JCI76262. little effect on cGMP and PKG activation (2). In contrast, conditions that induce or are associated with hypertrophic stress, including overexpression of the $G$ protein $\mathrm{Gq}$, transaortic constriction, or natriuretic peptide signaling, lead to increased sGC activity, subsequent cGMP production, and activation of PKG, which phosphorylates and activates a number of targets. PKG signaling is thought to reduce hypertrophic remodeling, and previous studies (3-5) have shown that PDE5 inhibition by drugs such as sildenafil can ameliorate the pathological cardiac remodeling that is associated with hypertrophy and heart failure (4-6). Conversely, overexpression of PDE5 in mice promotes pathological remodeling in the heart in the absence of cardiac stress $(3,7)$. Compared with tissue from healthy subjects, PDE5 expression is upregulated in patients with right ventricular failure (8). Furthermore, a small clinical trial demonstrated that silden- afil reduces pathological remodeling in patients with systolic heart failure (9); importantly, however, a large multicenter, placebo-controlled trial (RELAX) did not show a beneficial effect of sildenafil in the treatment of heart failure with preserved ejection fraction (10).

\section{Sex matters: sex-dependent responses to cardiac stress}

Although sex-associated differences are increasingly recognized in nonreproductive physiology, metabolism, and disease, these differences are generally not taken into account during clinical trial design or in drug treatment (11); however, this paradigm is beginning to change. For example, just last year the FDA recommended that women receive a different (lower) dose of zolpidem, a drug used to treat insomnia, than that recommended for men (12). In the FDA drug safety announcement about the new zolpidem dosing recommendations for women, it was noted that "women appear to be more susceptible to this risk (next morning impairment) because they eliminate zolpidem from their bodies more slowly than men." This example highlights that sex-dependent drug effects need to be taken into consideration when developing dosage guidelines.

There have been numerous sex-specific differences reported in cardiovascular physiology and disease (13). The majority of preclinical studies showing a beneficial effect of PDE5 inhibition on adverse remodeling were performed on male ani- 
A

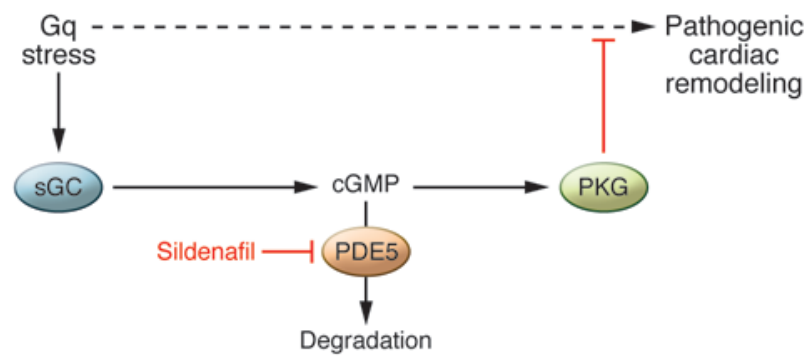

B

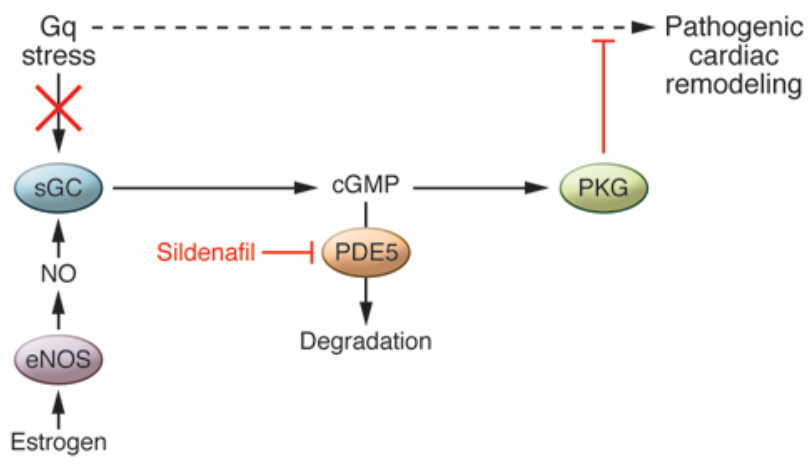

\section{Figure 1}

The cardioprotective effect of sildenafil in females is estrogen dependent. (A) In males, hypertrophic Gq stress activates SGC to promote the generation of cGMP, much of which is degraded by PDE5. Degradation of cGMP prevents the beneficial effects of PKG activation on pathological cardiac remodeling. Treatment of males with sildenafil inhibits PDE5, thus enhancing cGMP levels, which increases PKG activation. PKG phosphorylation of downstream targets blocks the pathogenic response to Gq stress. (B) In intact females, Gq stress promotes cardiac remodeling; however, $\mathrm{Gq}$ stress does not directly stimulate the generation of cGMP. Instead, estrogen is required to increases NO signaling, which leads to increased cGMP and activation of PKG. The protective effect of sildenafil in females requires the presence of estrogen to enhance cGMP and attenuate pathological remodeling. mals; therefore, it is worth considering that males and females may have different responses to PDE5 inhibition, and, if so, that the failure of the RELAX clinical trial, which included both males and females, may have been influenced by sex-specific effects. In this issue of the JCI, Sasaki et al. (14) directly addressed sex-dependent responses to PDE5 inhibition and examined the effect of sildenafil on hypertrophy-induced cardiac remodeling in female mice using Gq overexpression and pressure-overload models. In both hypertrophic stress models, sildenafil treatment did not reduce pathological remodeling in ovariectomized (OVX) females; however, sildenafil was protective against cardiac remodeling when OVX females received estrogen supplementation, indicating that sildenafil is only protective in females when estrogen is present (see Figure 1). Given that a substantial number of the female subjects in the RELAX trial were over the age of 50 , it is possible that many were postmenopausal with low estrogen levels, which might impair the beneficial effect of sildenafil on adverse remodeling. The results from Sasaki and colleagues reinforce the importance of studying the effects of treatments on both males and females in preclinical as well as in clinical trials. It is also important to perform preclinical studies on females with and without estrogen, as most cardiovascular disease occurs in older patients, which, for females, would translate to postmenopausal status.

\section{Estrogen and the female response to sildenafil}

The study by Sasaki et al. not only provides insight into an issue that possibly confounds the surprising results of the RELAX trial, but also raises a number of interesting questions regarding sexspecific differences in cardiac physiology and responses to sildenafil. Why is sildenafil beneficial for males, who naturally produce little estrogen, but not for females lacking or with reduced estrogen? Although the precise details for this difference will require further characterization, Sasaki et al. (14) demonstrated an approximately 50\% increase in PKG activity in male hearts from mice overexpressing $\mathrm{Gq}$ and that sildenafil administration further increased PKG activity in these animals. In contrast, $\mathrm{Gq}$ overexpression did not increase PKG activity in hearts from OVX females, and sildenafil treatment had no effect on PKG signaling; however, estrogen supplementation in OVX Gq-overexpressing females promoted an increase in PKG activation that was further increased with sildenafil, similar to what was observed in their male counterparts. Interestingly, under basal conditions, WT females exhibited higher PKG activity than did OVX females, and females overexpressing Gq had increased PKG activity that was enhanced following sildenafil treatment. Similar results were observed in mice that had undergone transaortic constriction to induce pressure overload; therefore, cardiac stress increased PKG in males (without estrogen), intact females, and OVX females supplemented with estrogen, but did not increase PKG activation in OVX females. Together, these results reveal that estrogen is required for the stress-induced elevation of PKG activity in females, but not in males. Interestingly, Sasaki et al. (14) also demonstrated that estrogen supplementation in male animals with cardiac stress increased PKG activity, reduced adverse remodeling, and was additive to the PKG-inducing effects of sildenafil.

These findings raise the question of why sildenafil is unable to activate PKG in females lacking estrogen, but is able to do so in males. In their report, Sasaki et al. speculate that in males, Gq activation leads to eNOS activation and increased cGMP, while in OVX females, eNOS is not activated. The precise details of this Gqmediated eNOS activation in males are unknown, but it has been speculated that androgens might contribute to stress-responsive activation of eNOS in males. Therefore, sildenafil inhibition of cGMP 
degradation by PDE5 further increases cGMP/PKG signaling and the beneficial effect of the drug in males. Conversely, females without estrogen do not exhibit activation of the eNOS/cGMP/PKG pathway; therefore, sildenafil has no beneficial effect when cGMP levels are not elevated in response to cardiac stress.

Future studies will be required to further elucidate whether the observed increase in PKG in males is due to androgens. The finding that PKG activation is lower in OVX females compared with that in males under cardiac stress, coupled with the reported beneficial effect of elevated PKG activity, suggests that remodeling is more severe in OVX females than in males. It will be interesting to examine the extent of cardiac remodeling in these populations in further detail and in other models of hypertrophic stress. It also appears as though there might be sex-related differences in PKG activity. Sasaki et al. (14) demonstrated that PKG activity was higher in intact females compared with that in OVX females, but their data also indicate that there was no difference in PKG activity between OVX females and males; therefore, it will be important to confirm this with a direct comparison. A sex-specific difference in PKG activity would be consistent with increased NOS activity in females.

\section{Conclusions}

In conclusion, this provocative study by Sasaki and colleagues adds to a growing body of evidence (11) that supports the analysis of clinical trial data by sex, rather than by simply adjusting for sex. It is clear that enhanced PKG signaling is beneficial in the setting of stress-induced heart failure and that there are important sexspecific differences in the signaling pathways that increase PKG activity. Moreover, sex-specific responses to cardiac stress can affect the response to drug treatment. The study by Sasaki et al. emphasizes the need for further examination of sex-dependent differences in cardiovascular function and disease and that differences between the sexes should be considered in the design of future clinical trials.

\section{Acknowledgments}

We acknowledge grant support ZO1HL-002065 and ZOI-HL-006059 to E. Murphy and RO1-HL-39752 to C. Steenbergen.

Address correspondence to: Elizabeth Murphy, Cardiac Physiology Section, Systems Biology Center, NHLBI, NIH, Bethesda, Maryland, USA. Phone: 301.496.5828; E-mail: murphy1@mail.nih.gov.

1. Lee DI, Kass DA. Phosphodiesterases and cyclic GMP regulation in heart muscle. Physiology (Bethesda). 2012;27(4):248-258.

2. Wallis RM, Corbin JD, Francis SH, Ellis P. Tissue distribution of phosphodiesterase families and the effects of sildenafil on tissue cyclic nucleotides, platelet function, and the contractile responses of trabeculae carneae and aortic rings in vitro. $A m J$ Cardiol. 1999;83(5A):3C-12C.

3. Vandenwijngaert $S$, et al. Increased cardiac myocyte PDE5 levels in human and murine pressure over- load hypertrophy contribute to adverse LV remodeling. PLoS One. 2013;8(3):e58841.

4. Nagayama T, et al. Sildenafil stops progressive chamber, cellular, and molecular remodeling and improves calcium handling and function in hearts with pre-existing advanced hypertrophy caused by pressure overload. J Am Coll Cardiol. 2009; 53(2):207-215.

5. Takimoto E, et al. Chronic inhibition of cyclic GMP phosphodiesterase $5 \mathrm{~A}$ prevents and reverses cardiac hypertrophy. Nat Med. 2005;11(2):214-222.

6. $\mathrm{Kim} \mathrm{KH}$, et al. Long-term effects of sildenafil in a rat model of chronic mitral regurgitation: benefits of ventricular remodeling and exercise capacity. Circulation. 2012;125(11):1390-1401.

7. Pokreisz P, et al. Ventricular phosphodiesterase-5 expression is increased in patients with advanced heart failure and contributes to adverse ventricular remodeling after myocardial infarction in mice. Circulation. 2009;119(3):408-416.

8. Shan X, Quaile MP, Monk JK, French B, Cappola TP, Margulies KB. Differential expression of PDE5 in failing and nonfailing human myocardium. Circ Heart Fail. 2012;5(1):79-86.

9. Guazzi M, Vicenzi M, Arena R, Guazzi MD. PDE5 inhibition with sildenafil improves left ventricular diastolic function, cardiac geometry, and clinical status in patients with stable systolic heart failure: results of a 1-year, prospective, randomized, placebocontrolled study. Circ Heart Fail. 2011;4(1):8-17.

10. Redfield MM, et al. Effect of phosphodiesterase-5 inhibition on exercise capacity and clinical status in heart failure with preserved ejection fraction: a randomized clinical trial. JAMA. 2013; 309(12):1268-1277.

11. Greiten LE, Holditch SJ, Arunachalam SP, Miller VM. Should there be sex-specific criteria for the diagnosis and treatment of heart failure? J Cardiovasc Transl Res. 2014;7(2):139-155.

12. Farkas RH, Unger EF, Temple R. Zolpidem and driving impairment - identifying persons at risk. NEngl J Med. 2013;369(8):689-691.

13. Murphy E. Estrogen signaling and cardiovascular disease. Circ Res. 2011;109(6):687-696.

14. Sasaki H, et al. PDE5 inhibitor efficacy is estrogen dependent in female heart disease. J Clin Invest. 2014;124(6):2464-2471. 